\title{
Size-controlled Growth of Fe Nanoparticles in Gas Flow Sputtering Process
}

\author{
H. Sakuma*, H. Aoshima, and K. Ishii \\ Department of Electrical and Electronic Engineering, Utsunomiya University, Utsunomiya 321-8585, Japan
}

(Received 22 March 2006)

\begin{abstract}
Iron (Fe) nanoparticles with diameters of $15-50 \mathrm{~nm}$ were obtained by the gas flow sputtering process. The particle size varied with the discharge current and Ar gas flow rate. While the particle size increased with the discharge current at an Ar flow rate of $500 \mathrm{sccm}$, the distribution of the particle diameters splits into two peaks for an Ar flow rate of $200 \mathrm{sccm}$ and low discharge current. At $200 \mathrm{sccm}$, particles of about $50 \mathrm{~nm}$ were obtained regardless of the discharge current; in addition, smaller particles with diameters depending on the discharge current were also obtained. The changes in the particle size are discussed in terms of Fe vapor density and growth period.
\end{abstract}

Key words : gas flow sputtering, Fe nanoparticle, particle size, particle size distribution

\section{Introduction}

Iron $(\mathrm{Fe})$ is the magnetic element that exhibits a fairly large magnetization. The nanoparticle of Fe also exhibits such properties in a restricted area of nanometer scale around the particle. Many applications of Fe nanoparticles have been proposed, e.g., in magnetic colloids [1], in magnetic sensors using the Hall effect [2], and as a catalyst for the growth of carbon nanotubes [3].

Fe nanoparticles are fabricated by using various dry and wet processes. The dry processes include thermal decomposition of organoiron [4] and evaporation of metal $\mathrm{Fe}$ [5]. Condensation of $\mathrm{Fe}$ atoms and their crystallization occurs in the gas phase, resulting in the formation of $\mathrm{Fe}$ nanoparticles. The wet processes include thermal decomposition of organoiron [6] and reduction of Fe ion. The Fe atoms that are formed coagulate and crystallize into $\mathrm{Fe}$ nanoparticles in the solution phase. The particle size is an important factor governing its magnetic properties. The difficulty in changing the particle size arises in some wet processes, while that in reducing particle size distribution arises in some dry processes. Surface covering matter such as surfactants and Fe oxides hides some properties of $\mathrm{Fe}$, e.g., electronic transport and catalytic properties. The surfactants are necessary for the wet process, and Fe oxides are observed in both the wet and dry processes.

*Corresponding author: Tel: +81-28-689-6104,

Fax: +81-28-689-6090, e-mail: hsakuma@cc.utsunomiya-u.ac.jp
Ishii et al. developed a dry process termed gas flow sputtering [7] for nanoparticle growth. In this process, the hollow cathode discharge in a tube target is utilized to obtain a high density of sputtered particles (atoms). The Ar sputtering gas, which is introduced at a fairly high pressure of about 1 Torr, doubles as a carrier gas. The high pressure makes the mean free path of the Ar and the sputtered particles as short as $0.1 \mathrm{~mm}$, and these particles lose their kinetic energy with collisions with Ar, resulting in the condensation. The Fe vapor density is variable with the sputtering power and the growth period is variable with the target-substrate spacing and gas flow rate; this can be advantageous in changing the particle size. In the previous study [7], Fe nanoparticles of 2-6 nm were obtained. However, it is necessary that Fe nanoparticles become larger than the superparamagnetic threshold of $12.5 \mathrm{~nm}$ [8] to exploit the ferromagnetic properties. In this study, the growth of Fe nanoparticles larger than the superparamagnetic threshold in a modified gas flow sputtering system is reported. In addition, the effect of changes in the discharge current and the Ar flow rate on the particle size and its distribution are reported, and the growth mechanisms are discussed.

\section{Gas Flow Sputtering System}

Figure 1 shows the gas flow sputtering system used in this study. A tube target with an inner diameter of $5 \mathrm{~mm}$ was used, and Ar gas was introduced in the chamber 


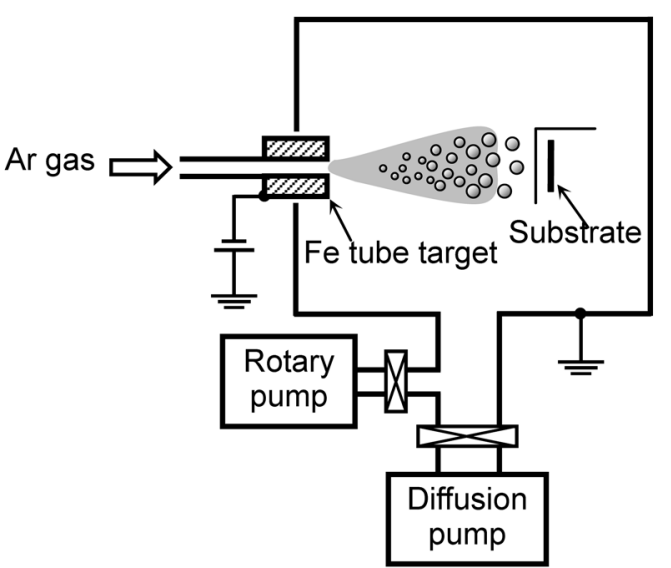

Fig. 1. Schematic diagram of the gas flow sputtering system.

through the target. In the previous study [7], a growth room was set in front of the target and $\mathrm{He}$ gas was introduced in the growth room. While this resulted in a complete growth of nanoparticles in the growth room, the particle size was as small as $2-6 \mathrm{~nm}$. The simpler set up in the present study is aimed at obtaining larger particles.

The chamber was evacuated to $3 \times 10^{6}$ Torr prior to sputtering and the pressure was maintained at 1 Torr during the sputtering. The spacing between the target and substrate was $17 \mathrm{~cm}$, and the distance of the substrate from the axis of target was $15 \mathrm{~mm}$. The DC voltage applied to the target was almost constant at $300 \mathrm{~V}$, regardless of the discharge current; therefore the sputtering power was proportional to the discharge current.

\section{Results and Discussion}

Fe nanoparticles were fabricated for several discharge currents and Ar flow rates. Figure 2 shows the transmission electron micrograph (TEM) images. The Fe nanoparticles were deposited for $3 \mathrm{~s}$ on substrated TEM grids. Fe nanoparticles larger than $10 \mathrm{~nm}$ were formed at all the conditions. In addition to such particles, smaller particles of about $3 \mathrm{~nm}$ and thin-film-like matter were formed at an Ar flow rate $F=500 \mathrm{sccm}$ and high discharge currents. These originated from $\mathrm{Fe}$ atoms or clusters which were not condensed into nanoparticles. All the large nanoparticles acquire an $\mathrm{Fe} / \mathrm{Fe}$-oxide core/shell structure, and the thickness of the shell is about $2 \mathrm{~nm}$. The electron diffraction pattern shown in the lowest panel of Fig. 2 is that of a nanoparticle fabricated at a discharge current $I_{d}=0.8 \mathrm{~A}$ and $F=200 \mathrm{sccm}$ (shown in the upper panel). The diffraction spots indicate that the core of the particle is a single crystal of Fe. The weak rings originate

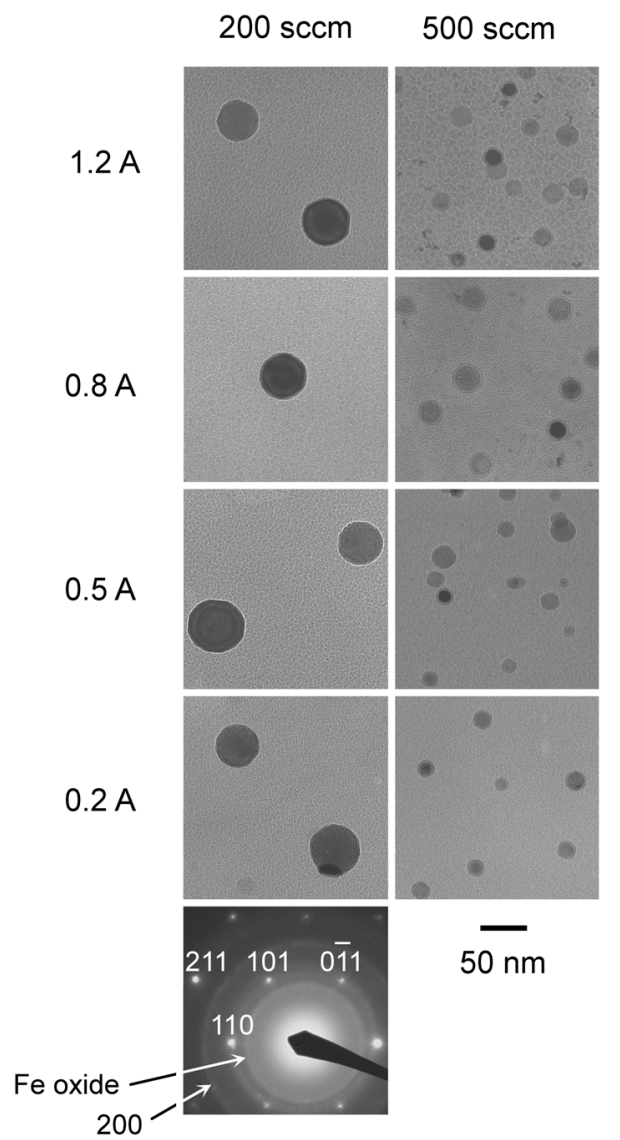

Fig. 2. TEM images of Fe nanoparticles obtained at discharge currents of 0.2-1.2 A and Ar flow rates of 200 and $500 \mathrm{sccm}$. The electron diffraction pattern is of the Fe nanoparticle shown in the upper panel at $0.8 \mathrm{~A}, 200 \mathrm{sccm}$. The indexes are of $\alpha$ Fe.

from $\mathrm{Fe}$ oxide in the shell and $\mathrm{Fe}$ in the thin-film-like matter around the particle.

Particle size distribution was obtained by TEM observation and is shown in Fig. 3. The curves are a result of curve-fitting by the lognormal function [9]:

$$
f(D)=\frac{1}{D \sigma \sqrt{2 \pi}} \exp \left[-\frac{1}{2}\left(\frac{\ln D-\mu}{\sigma}\right)^{2}\right],
$$

where $D$ is the particle size, and $\sigma$ and $\mu$ are fitting coefficients. The expectation value and standard deviation of the lognormal distribution, $E(D)$ and $S(D)$ are expressed as

$$
E(D)=A \sqrt{B}
$$

and

$$
S(D)=A^{2} B(B-1),
$$

where $A=\exp \mu$ and $B=\exp \sigma^{2}$. Each distribution has a 


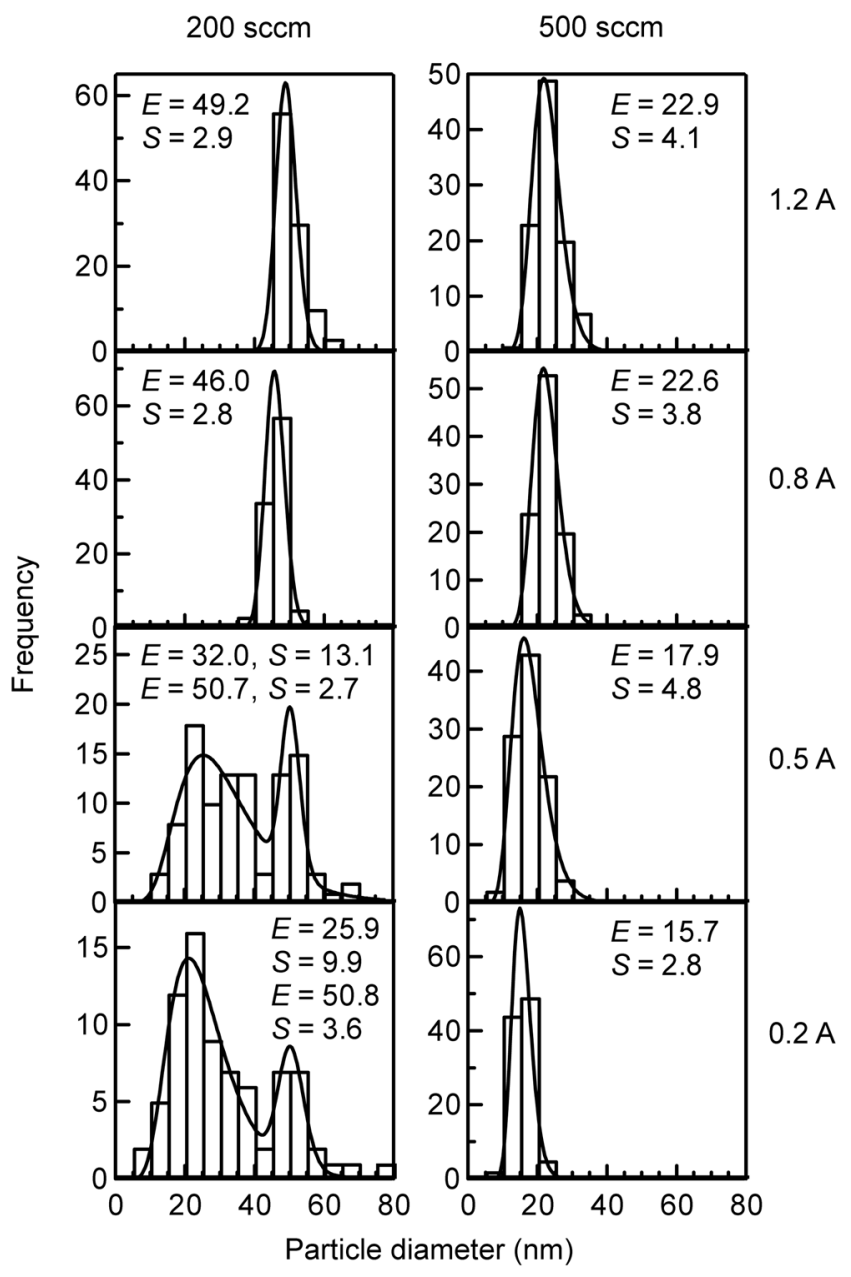

Fig. 3. Distribution of particle diameters obtained by TEM observation. The curves are results of the curve-fitting by the lognormal function. $E$ and $S$ are expectation value (nm) and standard deviation (nm) for the lognormal distribution, respectively.

fairly sharp peak at around $50 \mathrm{~nm}$ for $F=200 \mathrm{sccm}$ and at around $20 \mathrm{~nm}$ for $F=500 \mathrm{sccm}$. The distribution for $I_{d}$ $=0.2$ and $0.5 \mathrm{~A}$ and $F=200 \mathrm{sccm}$ has another broad peak at about $20 \mathrm{~nm}$. The curves were fitted as a superposition of two lognormal functions. Figure 4 shows a plot of the particle diameter (the expectation value of the distribution) against the discharge current. The particle diameter monotonically decreases with the discharge current for $F$ $=500 \mathrm{sccm}$, while the distribution splits into two peaks at $I_{d}=0.8$ A for $F=200 \mathrm{sccm}$.

Figure 5 shows the magnetization curve of $\mathrm{Fe}$ nanoparticles measured at room temperature and the sweep rate of magnetic field of $67 \mathrm{Oe} / \mathrm{s}$. About one layer of $\mathrm{Fe}$ nanoparticles were deposited on a glass substrate at $I_{d}=$ $0.8 \mathrm{~A}$ and $F=200 \mathrm{sccm}$. The magnetization almost saturated at $10 \mathrm{kOe}$, indicating that the proportion of

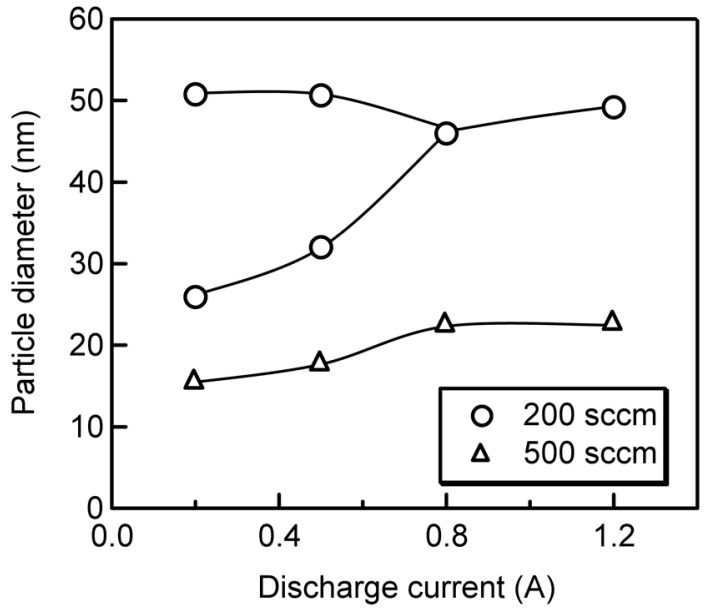

Fig. 4. Particle diameter (expectation value) as a function of the discharge current.

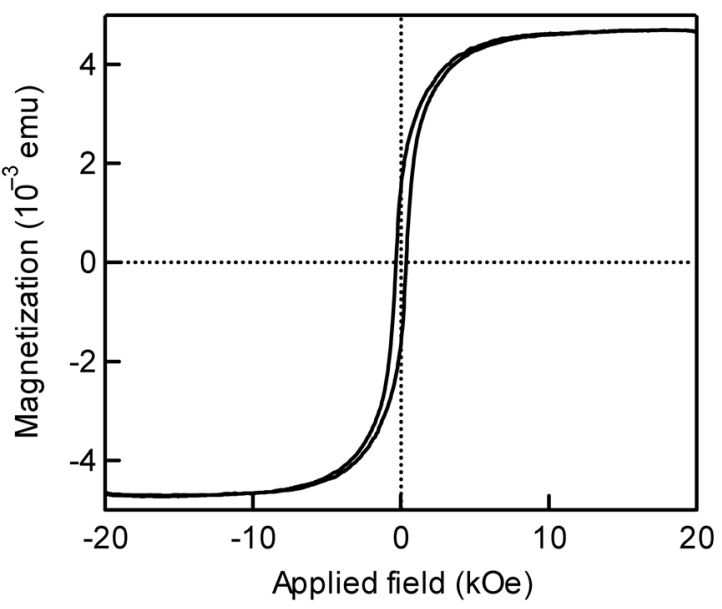

Fig. 5. Magnetization curve of Fe nanoparticles obtained at a discharge current of $0.8 \mathrm{~A}$ and an Ar flow rate of $200 \mathrm{sccm}$. The measurements were carried out at room temperature.

superparamagnetic particles is small. The coercivity was $340 \mathrm{Oe}$, which is lower than the reported values, e.g., 770 Oe for Fe nanoparticles with a diameter of $30 \mathrm{~nm}$ [5] and 650 Oe for those with a diameter of $15 \mathrm{~nm}$ [10]. The low coercivity may be ascribed to the domain wall motion. The coercivity was higher for a faster sweep rate of magnetic field of $130 \mathrm{Oe} / \mathrm{s}$ : $490 \mathrm{Oe}$, which means that the magnetization reversal with thermal activation is not negligible. While the coercivity is about 500 Oe (at 130 $\mathrm{Oe} / \mathrm{s}$ ) and slightly increases with the discharge current for $500 \mathrm{sccm}$, it is the greatest (490 Oe) at $0.8 \mathrm{~A}$ for 200 sccm. Therefore, the particle size is not a decisive factor for coercivity; other factors should be taken into account. For example, inter-particle interaction, which varies with the packing density of the particles, may have an effect on the coercivity. 


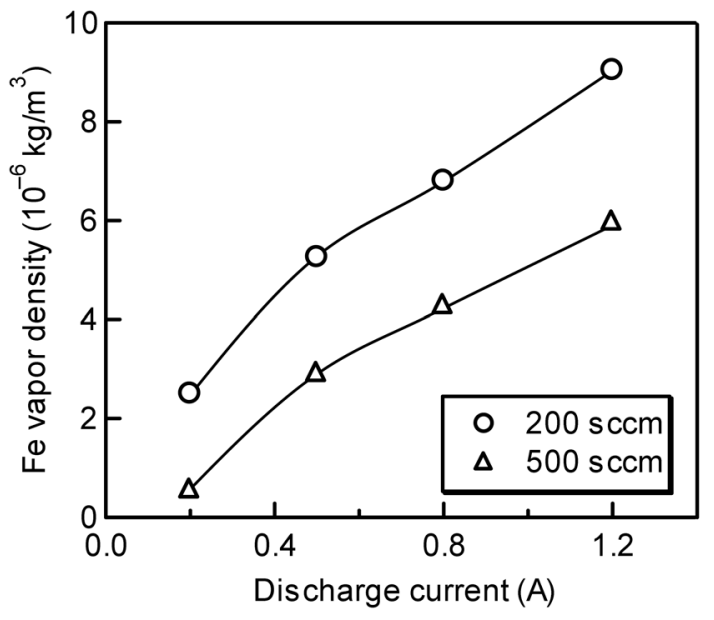

Fig. 6. Fe vapor density as a function of the discharge current.

We suppose that the growth mechanism of Fe nanoparticles in the gas flow sputtering process is as follows. Fe atoms are generated from the target by the sputtering process at 1 Torr, and supersaturated Fe vapor is produced. Fe atoms collide with each other and homogeneous nucleation occurs. The growth of a nucleus proceeds with the coagulation of nuclei or the adsorption of $\mathrm{Fe}$ atoms on the nucleus. On the basis of the above supposition, the Fe vapor density is an important factor that governs the particle size. Thus, we conducted the measurement of Fe vapor density as follows. The mass of Fe released from the target was estimated by measuring the mass of the deposit on a substrate set close to it using inductively coupled plasma spectroscopy. Further, the volume of gas blowing out from the target was estimated with assumptions of a gas temperature of $100{ }^{\circ} \mathrm{C}$ and a gas pressure of 1 Torr. From these two values-the mass of $\mathrm{Fe}$ and the volume of gas - the Fe vapor density is estimated. Figure 6 shows the relationship between the estimated Fe vapor density and discharge current. For both Ar flow rates, the $\mathrm{Fe}$ vapor density monotonically increased with discharge current. The Fe vapor density for $F=200 \mathrm{sccm}$ is higher than that for $F=500 \mathrm{sccm}$.

The particle diameter (the expectation value of particle size distribution) is replotted as a function of $\mathrm{Fe}$ vapor density in Fig. 7. The data appear to be classified into two components: one that is dependent on and the other that is independent of $\mathrm{Fe}$ vapor density. The vapor-densitydependent component is interpreted as follows. The probability of collision of Fe atoms or nuclei becomes higher as the vapor density increases. Since the growth of nanoparticles increases with the collision frequency, larger particles are obtained in higher vapor densities.

The vapor-density-independent component, which corresponds to the particle obtained at $F=200 \mathrm{sccm}$, should

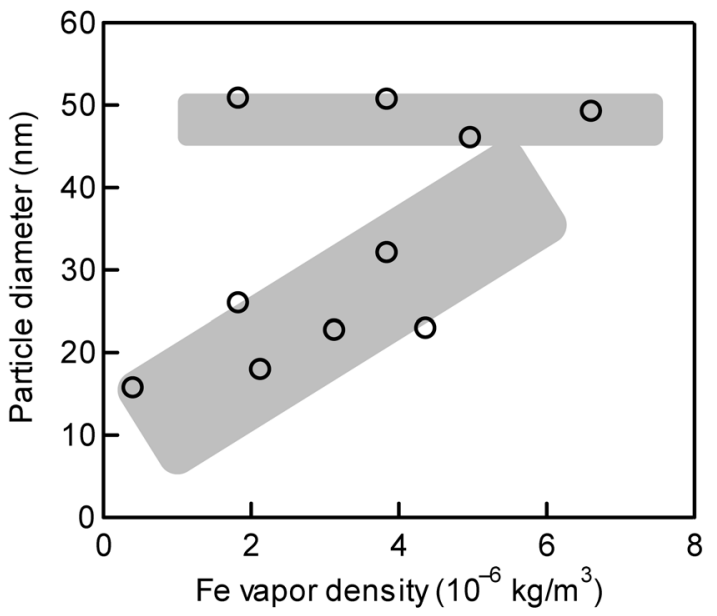

Fig. 7. Particle diameter (expectation value) as a function of the Fe vapor density.

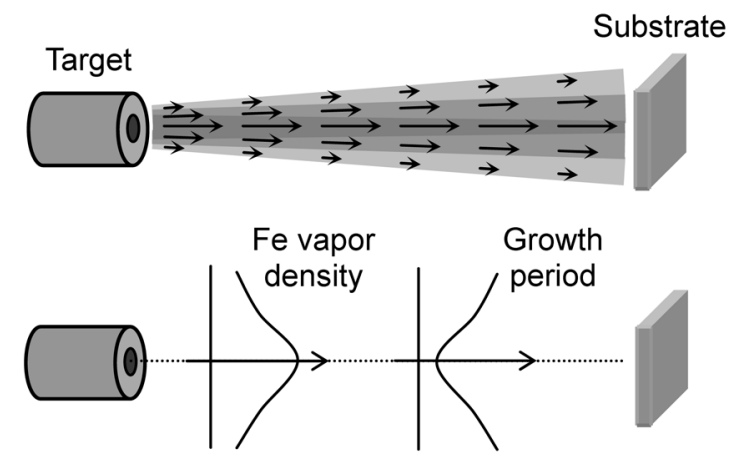

Fig. 8. Schematic diagram of changes in Fe vapor density and growth period with distance from the axis of target.

be ascribed to a different factor. The growth period is another important factor: The particle size becomes larger as the growth period becomes longer. Figure 8 shows a schematic diagram explaining the distribution of Fe vapor density and growth period. The velocity of gas flow is the highest on the axis of the target and decreases with distance from the axis, therefore the growth period increase with distance from the axis. The increase in particle size with distance from the target axis was observed experimentally, and it is shown in Fig. 9. The sputtering was performed with target inner diameter of $4 \mathrm{~mm}, I_{d}=1.2 \mathrm{~A}$, $F=500 \mathrm{sccm}$, and deposition time was $1 \mathrm{~s}$. Fe particles with diameter of about $10 \mathrm{~nm}$ were deposited in both positions, whereas in the off-axis position, larger particles over $20 \mathrm{~nm}$ were observed in addition to the small particles. However, on the contrary, the Fe vapor density decreases with distance from the target axis. The change in particle size with distance from the target axis is determined by the balance of the Fe vapor density and growth period (velocity of gas flow). If the growth period 


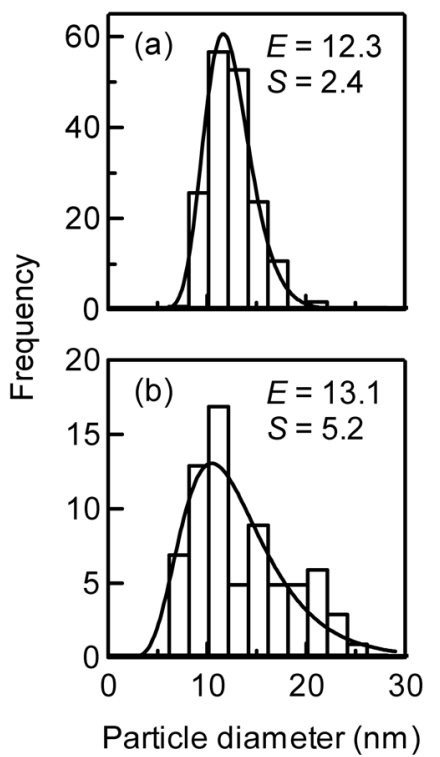

Fig. 9. Change in particle size distribution with distance from the axis of target. (a) On the axis, and (b) $15 \mathrm{~mm}$ from the axis. The meaning of the lines and the symbols are the same as Fig. 3.

is sufficiently long, the particle size may attain a kind of equilibrium [11]. As the growth of particle proceeds, the concentration of $\mathrm{Fe}$ atoms, which is necessary for the particle growth, becomes low, and finally the particle growth terminates. The vapor-density-independent component may correspond to the Fe nanoparticles with an equilibrium particle size.

The particle size distribution for low rate of gas flow and low discharge current split into two peaks. The Fe vapor density is low because of the low discharge current and the growth period is long because of the low rate of gas flow. In this situation, the equilibrium particle size may be attained, and equilibrium large particles and small particles, which are inhibited further growths for the low concentration of $\mathrm{Fe}$ atoms, may coexist.

In the previous study [7], a growth room was set in front of the target and $\mathrm{He}$ gas was introduced. Large particles were expected, since the growth of nanoparticles could be completed in the vigorous turbulent flow. How- ever, in reality the obtained particles were small. This was ascribed to a low $\mathrm{Fe}$ vapor density since the $\mathrm{He}$ gas dilutes the $\mathrm{Fe}$ vapor. However, a detailed calculation or a simulation like the aerosol dynamics simulation is necessary to obtain conclusive evidence.

\section{Conclusion}

Fe nanoparticles with diameters of $15-50 \mathrm{~nm}$ can be obtained by using a simple gas flow sputtering system. While the particle size increases with the discharge current at the Ar flow rate of $500 \mathrm{sccm}$, the distribution of the particle diameters splits into two peaks at $200 \mathrm{sccm}$ and low discharge current. The $\mathrm{Fe}$ vapor density and growth period may have a profound effect on the particle size.

\section{References}

[1] X. X. Zhang, B. X. Qin, G. H. Wen, Y. S. and Kwok, K. K. Fung, Mater. Sci. Eng. C 16, 119 (2001).

[2] Y. Li, P. Xiong, S. von Molnár, S. Wirth, Y. Ohno, and H. Ohno, Appl. Phys. Lett. 80, 4644 (2002).

[3] W. Z. Li, S. S. Xie, L. X. Qian, B. H. Chang, B. S. Zou, W. Y. Zhou, R. A. Zhao, and G. Wang, Science 274, 1701 (1996).

[4] B. Giesen, H. R. Orthner, A. Kowalik, and P. Roth, Chem. Eng. Sci. 59, 2201 (2004).

[5] A. Tasaki, S. Tomiyama, S. Iida, N. Wada, and R. Uyeda, Jpn. J. Appl. Phys. 4(10), 707 (1965).

[6] D. Farrell, S. A. Majetich, and J. P. Wilcoxon, J. Phys. Chem. B 107(40), 11022 (2003).

[7] K. Ishii, K. Amano, and H. Hamakake, J. Vac. Sci. Technol. A 17(1), 310 (1999).

[8] K. Ohta, Basis of Magnetics II, Kyoritsu, Tokyo (1973), pp. 336 (in Japanese).

[9] S. Muto, Handbook of Statistical Analysis, Asakura, Tokyo (1995), pp. 131 (in Japanese).

[10] A. Slawska-Waniewska, A. Roig, M. Gich, Ll. Casas, K. Racka, N. Nedelko, and E. Molins, Phys. Rev. B 70, 054412 (2004).

[11] K. Takahashi, Basis of Aerosol Dynamics, Morikita, Tokyo (2003), chap. 6 (in Japanese). 